\title{
Per 2500 White Blood Cells
}

National Cancer Institute

\section{Source}

National Cancer Institute. Per 2500 White Blood Cells. NCI Thesaurus. Code 132474.

Natural number unit of measurement for a portion of a particular type of cell (excluding white blood cell subtypes) per 2500 white blood cells. 